\title{
Online Peer Support Groups for Behavior Change: Moderation Requirements
}

\author{
Manal Aldhayan ${ }^{1}$, Mohammad Naiseh ${ }^{1}$, John McAlaney ${ }^{1}$, Raian Ali ${ }^{2}$ \\ ${ }^{1}$ Bournemouth University, UK \\ \{maldhayan, mnaiseh, jmcalaney\}@bournemouth.ac.uk \\ ${ }^{2}$ College of Science and Engineering, Hamad Bin Khalifa University, Qatar \\ raali2@hbku.edu.qa
}

\begin{abstract}
Technology-assisted behaviour awareness and change is on the rise. Examples include apps and sites for fitness, healthy eating, mental health and smoking cessation. These information systems recreated principles of influence and persuasion in a digital form allowing real-time observation, interactivity and intervention. Peer support groups are one of the behavioural influence techniques which showed various benefits, including hope installation and relapse prevention. However, unmoderated groups may become a vehicle for comparisons and unmanaged interactions leading to digression, normalising the negative behaviour and lowering self-esteem. A typical requirement of such groups is to be of a social and supportive nature whereas moderation, through humans or artificial agents, may face a risk of being seen as centralised and overly managed governance approach. In this paper, we explore the requirements and different preferences about moderators as seen by members. We follow a mixed-method approach consisting of a qualitative phase that included two focus groups and 16 interviews, followed by a quantitative phase, including a survey with 215 participants who declared having well-being issues. We report on the qualitative phase findings achieved through thematic analysis. We also report and discuss the survey results studying the role of gender, self-control, personality traits, culture, the perception of usefulness and willingness to join the group as predictors of the members' expectations from moderators, resulted from the qualitative phase.
\end{abstract}

Keywords: Human factors in information systems, Peer support groups, Behaviour change system

\section{$1 \quad$ Introduction}

There is a growing number of studies on the use of technology to combat problematic behaviour and enhance wellbeing. Examples include the use of mobile apps for smoking cessation, improving mental health, fitness, diet and physical activities [21]. The advances in sensing technology and handheld devices combined with the ubiquitous connectivity to internet created opportunities for utilising technology to assist behavioural change and self-regulation systems in a more intelligent, contextualised and situation-aware style. Such solutions have been applied both in work environments, e.g. gamifying task performance [29], and in a personal context such as enhancing wellbeing and combatting problematic behaviour $[19,20]$. 
Most technology-assisted behaviour awareness applications available in the market and discussed in the literature are meant for a single user where the communication is between the software and the user and where exploitation of social techniques, such as peer learning and support, is limited. The main challenge of these information systems was typically to engage users with such solutions and increase their retention [33]. For example, Ciocarlan et al. [28] studied and designed an application around happiness where user engagement was maximised by sending different persuasive messages for activities to do. Feedback messages about individual performance are the main techniques utilised in health applications to motivate users and keep them engaged [24].

The strategies and techniques used to motivate, and influence behaviour can differ according to the personal and environmental context. Factors such as age, personality traits, gender and culture have been studied, and such differences were identified. Orji and Mandryk [25] studied the effect of culture on the persuasive intervention in the context of healthy eating behaviour change applications, as well as the role of gender and age groups as moderating factors. Also, Orji et al. [26] studied the effect of gender and age on the six principles of influence proposed by Cialdini [30] and showed significant differences. Mainly, it showed that females are more responsive to most of the influence strategies than males. Alkis and Temizel [27] studied the relationship between personality traits and the effectiveness of Cialdini strategies and showed significant differences. For example, people with high agreeableness (as one of the Big Five personality traits model [31]) are more likely to be affected by the opinions of others whether peer, i.e. social proof, or authority (two of Cialdini strategies [30]).

Online peer groups are a type of technology-assisted behaviour awareness software that is meant to provide peer support, counselling, motivational and learning environment, and ambivalence reduction through sharing and hope installation. Online peer groups are a synthesis of various influence strategies, including peer pressure, commitment and goal setting, surveillance, and authority through moderator or caregiver. This means that online peer groups, in their governance, design, acceptance and rejection can similarly be affected by variables like gender, personality traits, culture and selfcontrol. Peer groups are typically moderated to prevent unintended harmful interactions within them. They may become a forum for learning or boosting negative behaviours and normalising the problematic behaviour and reducing the sense of culpability of committing it due to excessive peer emotional support [22]. However, the moderator role is delicate as the spirit of groups is social and authority can be seen as overly restrictive and deter members from joining, entice reactance, conformity and dishonesty.

In this paper, we explore the peer groups members perception of the moderator role and how their personal and cultural characteristics can affect that perception. This is to help the engineering of online peer groups platforms so that moderators are assisted by tools and access needed to play their role and also to help the governance strategies and configuration of such online platforms. As a method, we adopted a mixed-method approach exploring in its qualitative phase the members' perception of the role of the moderator and in its quantitative face, the effect of gender, self-control, personality traits, culture and the perception of usefulness and willingness to join the group on that perception. Such users' studies yield important knowledge for health and social information systems design and increasing users' acceptance [34, 35]. 


\section{Research Method}

We adopted a mixed-methods approach which consisted of an initial qualitative phase followed by a quantitative phase. The participants in both phases self-declared to have problematic behaviour, mainly online behaviour, that has been affecting their wellbeing issues.

\subsection{Qualitative phase: exploring participants perception of the moderator role}

We first conducted a focus group study consisting of two sessions. The first session aimed at getting insights into how online peer groups are perceived by people who selfdeclared to have problematic online behaviour and what they needed to see in it. The second focus group aimed at identifying the design of online peer groups platforms where mock interfaces were made available to the participants. The interfaces were based on the results of the first focus group, and the participants were able to amend and comment on them. The two focus group sessions were conducted with the same six university students; three male and three females, aged between 20 and 26. The participants were a social group in real life, and this was beneficial as it removed concerns regarding trust and privacy during the discussion process. We performed a thematic analysis [23] on the data collected through the sessions and analysed the annotations on the interfaces. The analysis revealed main factors concerning the (i) acceptance and (ii) rejection factors of this approach as well as (iii) governance styles and (iv) moderator profile and role.

Then we performed in-depth interviews to delve into the details of these themes. For example, we explored the role of feedback and monitoring, membership and exit protocol within the governance theme and the skills expected, allocation strategy and authority within the moderator role. We conducted 16 interviews with students who selfdeclared to have a wellbeing issue around their digital behaviour, e.g., obsessive or compulsive use. The sample consisted of 8 males and 8 females, aged between 18 and 35 . Each interview lasted between 30 and 40 minutes. The interviews were transcribed and analysed via thematic analysis [23].

\subsection{Quantitative stage: members' profile effect on perception of moderator}

We designed a survey around the interview findings, which included the perception of moderator and their role.The survey was disseminated both online and in person. A $£ 5$ incentive was offered to respondents given the lengthy nature of the survey. We collected 215 completed responses; 105 (49\%) male and 109 (50\%) Female and one participant preferred not to answer on the gender question. The participants were 17 to 55 years old. The survey started with a validation question of whether a participant has wellbeing issues as a precondition to take part.

To study the effect of personal and environmental factors on the perception of moderator, the survey included questions around six factors which were gender (male/ female); country; perceived usefulness of peer support groups; willingness to join a peer 
support group; the five personality traits [31] (extraversion, agreeableness, conscientiousness, neuroticism and openness); and self-control [32]. The survey included 29 questions around the six themes of moderator roles which were the findings from the qualitative study, summarised in Table 1 and Table 2. The moderator roles Likert scale questions are based on "agreeing" or "disagreeing" with five rating scale. We disseminated the survey mainly in the UK, the Kingdom of Saudi Arabia (KSA) and Syria. We collected 104 completed surveys from KSA and Syria (55 male/ 49 female, mean age $=26.7, \mathrm{SD}=6.39)$, and 85 from the UK ( 35 male/ 50 female, mean age $=24.07, \mathrm{SD}=$ 6.39) while the rest were from other countries, mainly in Europe. This allowed us to study statistically whether there was a difference between Middle Eastern culture (KSA and Syria) and Western culture (UK). As such, the total sample size used within the analysis reported here was 189 .

\section{Moderation requirements: members perspective}

The qualitative phase analysis revealed six main themes are summarised in Figure 1and Figure 2. Table 1 and Table 2 include the phrasing in survey question which was used to reflect the qualitative findings. In the survey, we aggregated codes further to reduce the number of survey options.

Figure 1. Moderator profile and role as seen by members: thematic map

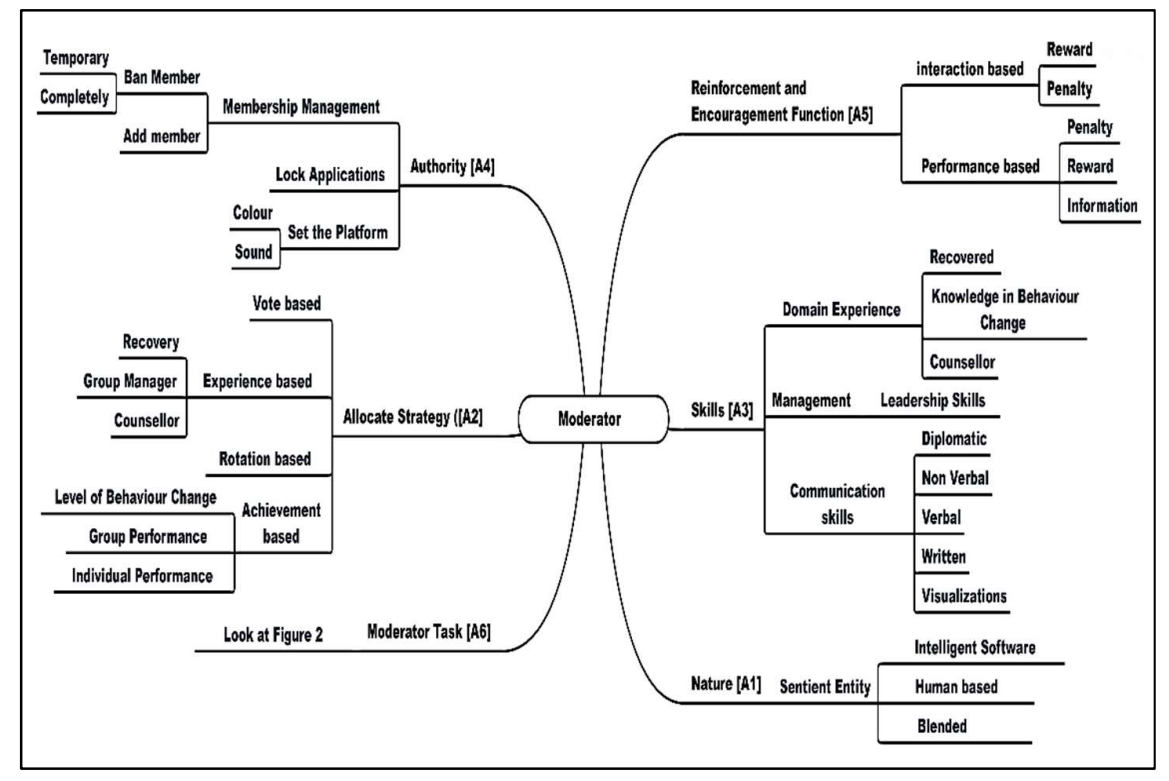


Table 1. Moderator profile and role as seen by members: a table view

\begin{tabular}{|c|c|}
\hline Theme & Sub-theme \\
\hline $\begin{array}{l}\text { [A1] } \\
\text { Moderator nature }\end{array}$ & $\begin{array}{l}\text { [A1.1] Software, e.g. automatic target calculation and advice-giving } \\
\text { [A1.2] Human } \\
\text { [A1.3] Blended, i.e. human and software together }\end{array}$ \\
\hline $\begin{array}{l}{[\mathrm{A} 2]} \\
\text { Moderator alloca- } \\
\text { tion strategy }\end{array}$ & $\begin{array}{l}\text { [A2.1] Voting by members } \\
\text { [A2.2] Experience-based, e.g. experience in group management, } \\
\text { counselling, previous success, etc. } \\
\text { [A2.3] Rota-based, i.e. each member becomes a moderator for some- } \\
\text { time } \\
\text { [A2.4] Performance, e.g. those who prove to be a helper to others, en- } \\
\text { hancing personal wellbeing score, etc. }\end{array}$ \\
\hline $\begin{array}{l}{[\mathrm{A} 3]} \\
\text { Moderator skills }\end{array}$ & $\begin{array}{l}\text { [A3.1] Had the well-being issue in the past and recovered from it } \\
\text { [A3.2] High communication skills } \\
\text { [A3.3] Management and leadership skills }\end{array}$ \\
\hline $\begin{array}{l}{[\text { A4] }} \\
\text { Moderator author- } \\
\text { ity }\end{array}$ & $\begin{array}{l}\text { [A4.1] To manage membership, e.g. adding new members and ban- } \\
\text { ning members who violate the rules } \\
\text { [A4.2] To ban members from doing certain activities, e.g. banning } \\
\text { video games and social media at night hours } \\
\text { [A4.3] To set up the online environment, e.g. the colours, the forum } \\
\text { topics, the sounds, the reminders }\end{array}$ \\
\hline $\begin{array}{l}\text { [A5] } \\
\text { Moderator rein- } \\
\text { forcement role }\end{array}$ & $\begin{array}{l}\text { [A5.1] Reward members based on the improvement of their perfor- } \\
\text { mance } \\
\text { [A5.2] Issue penalty based on the poor performance } \\
\text { [A5.3] Reward members based on interactions, e.g. help others and } \\
\text { adherence to chat rules } \\
\text { [A5.4] Issue penalty based on interactions within the online group }\end{array}$ \\
\hline
\end{tabular}

Figure 2. Moderator tasks as seen by members: thematic map

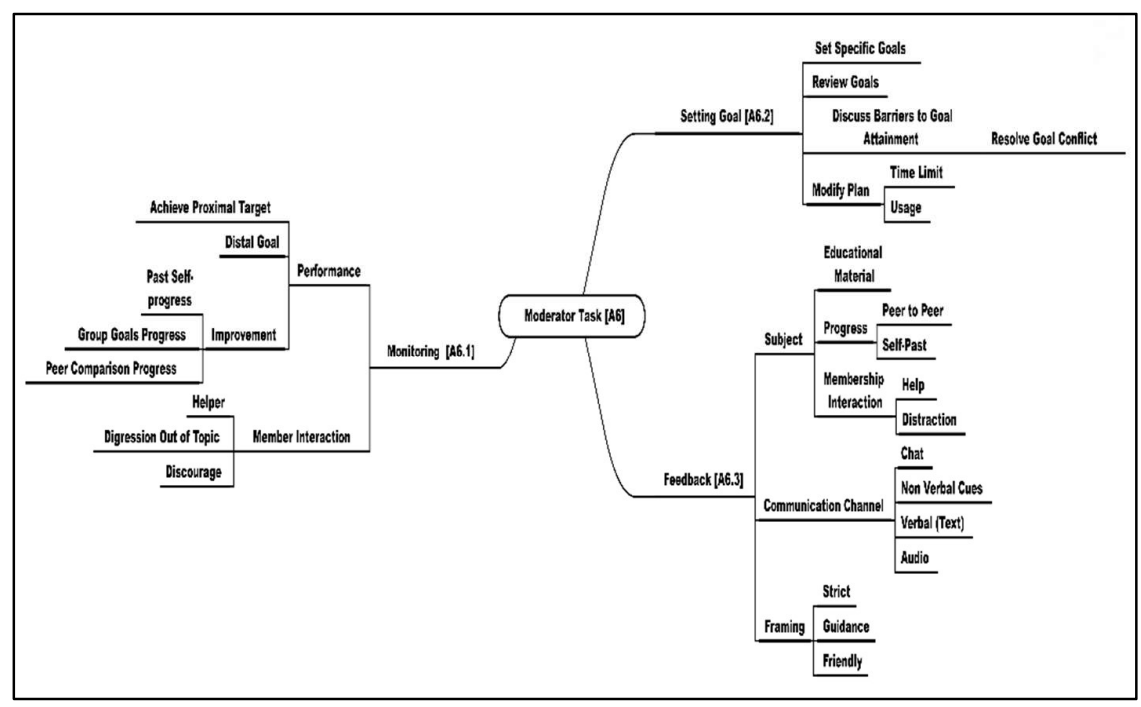


Table 2. Moderator tasks as seen by members: a table view

\begin{tabular}{|c|c|}
\hline Theme & [A6] Moderator tasks \\
\hline [A6.x] Sub-theme & [A6.x.y] Sub-Sub-theme \\
\hline $\begin{array}{l}\text { [A6.1] } \\
\text { Monitor group } \\
\text { members }\end{array}$ & $\begin{array}{l}\text { [A6.1.1] Access the data about members' performance, e.g. achieve- } \\
\text { ment of goals and progress made towards them. } \\
\text { [A6.1.2] Access data around the style of communication of members, } \\
\text { e.g. reports indicating members to be helpful, distractor, digression, } \\
\text { etc. }\end{array}$ \\
\hline $\begin{array}{l}\text { [A6.2] } \\
\text { Manage perfor- } \\
\text { mance goals }\end{array}$ & $\begin{array}{l}\text { [A6.2.1] Specify performance goals for members } \\
\text { [A6.2.2] Modify goals for members, e.g. grant an extension } \\
\text { [A6.2.3] Review goal achievement with members frequently } \\
\text { [A6.2.4] Discuss barriers to goals achievement with members, e.g. re- } \\
\text { solving conflicting goals. } \\
\text { [A6.2.5] Send personalised best practices and advice on how to } \\
\text { achieve goals to members }\end{array}$ \\
\hline $\begin{array}{l}\text { [A6.3] } \\
\text { Provide feedback }\end{array}$ & $\begin{array}{l}\text { [A6.3.1] Send feedback about how the group is performing as a } \\
\text { whole, i.e. collectively } \\
\text { [A6.3.2] Send feedback about self-progress to members, e.g. their } \\
\text { self-improvement } \\
\text { [A6.3.3] Send feedback to members about their interaction, e.g. being } \\
\text { seen as a helper or distractor } \\
\text { [A6.3.4] Choose the communication channel to use with members, } \\
\text { e.g. text, audio, non-verbal such as emoji, chat, etc. } \\
\text { [A6.3.5] Choose the framing and the tone of the feedback, e.g. guid- } \\
\text { ance, assertive, strict, friendly, etc. }\end{array}$ \\
\hline
\end{tabular}

\subsection{Moderator Nature}

The analysis indicated various requirements of the group moderator's traits, especially of the sentient nature of the moderator. Participants have three viewpoints regarding the moderator's nature. The first viewpoint [A1.1] was that the platform should be managed by intelligent software which would provide "24-hour help, and advice and members will get a response immediately from the moderator". The second viewpoint [A1.2] was that the moderator should be human and that "would help to understand members feelings and provide support based on a human experience". The last viewpoint [A1.3] recommended that the platform is designed to have blended management which has both human and software management. The participants argued that the platform should be assisted by intelligent software and administered and configured by a human moderator for tailoring, scheduling and sending notifications and feedback messages. Participants emphasised that human moderator is more credible for providing emotional support and personalised dialogue.

\subsection{Moderator Allocation Strategy}

There has not been a consensus on the strategy to allocate moderator and different viewpoints were expressed. The first opinion [A2.1] was that moderator allocation is 
done based on voting technique and "group members should vote for the group moderator, the moderator could be a group member, or persons offer themselves to be moderator". The second opinion [A2.2] is that moderator allocation should be based on personal experiences such as having the same problem as the members in the past or skills in group management and counselling. The third opinion [A2.3] is that the moderator allocation should be a rota-based and everyone is given a chance to play it as this "would help members to learn how to be moderator" and also increase their belongingness and relatedness to others. The last opinion [A2.4] is that group moderators should be one of the group members and allocated based on the past performance and goals achieved. A participant commented that "the member who has achieved most of the group goals or collected more points could become the moderator of the group" and act as a role model.

\subsection{Moderator Skills}

The facet of moderator knowledge and experience was highlighted as one of the most important factors which would motivate members to join a group and engage in its interactions. Different requirements were expressed. The first [A3.1] was domain experience, such as having had the same issue in the pasting and overcoming it so that their advice and support are more influential. The second [A3.2] related to having communication skills including verbal, non-verbal, written, visualizations and diplomatic skills. The participants justified the need for diplomatic skills believing that this would help members epically when they set collective goals requiring all of them to work together and also help to manage the members' interactions and potential tensions. The third [A3.3] related to leadership and management skills. The participants felt that the moderator who has leadership skills would be able "to explain in clear steps how to achieve the goals" and what is expected for them to do so and be able to follow up.

\subsection{Moderator Authority}

It was generally agreed that moderators should have the power to manage group membership, restrict some interactions and actions and change the platform interface. The first authority aspect [A4.1] related to managing membership, such as adding members and keeping groups live so that "if a member leaves the group the group moderator should have the ability to replace them with a new member". The second [A4.2] related to the ability to ban members based on progress or unhealthy and negative interactions with others. A participant commented that "any member who doesn't interact with other group members could be banned for a period of time by the moderator" as this is against the spirit of being in a group. Others suggested that "any member who distracts other members could be banned". The third moderator authority aspect [A4.3] related to the manager ability to change the online platform settings such as the colours, available emoji and notification sounds. Participants mentioned that this could be for cosmetics purposes but also to reflect progress, e.g. the use of colours to indicate group member performance and collective performance. 


\subsection{Reinforcement and Encouragement Functions}

Participants felt that the online peer groups should provide tools for the moderator to apply reinforcement and encouragement functions around both the performance towards achieving goals and the interactions with other members. The moderator shall be able to reward [A5.1] and issue penalty [A5.2] based on tracking and comparing member performance in relation to achieving goals. Similarly, the moderator shall be able to give rewards [A5.3] and issue penalty [A5.4] based on the characteristics of the interactions of a member within the group, e.g. being helpful, adhering to the chat rules, and avoiding distraction and private messaging outside the group. A user who becomes a helper, for example, by helping other group members with advice and moral support should "get points and a member who has high points could become group admin as a reward". Penalty, such as reducing points or banning members, temporarily or completely should be related to when "violating the group norms and disturbing others". The participants appreciated that the group moderator should be able to monitor the group members' progress and performance toward their goals and interactions. Also, the participants thought members performance and interaction shall not be measured in a uniform style as members roles and stage of behaviour change can be different.

\subsection{Moderator Tasks}

The group moderator is considered central to running and managing the online peer groups. The participants suggested various preferences and opinions around the set of activities and tasks for moderators which are [A6.1] Monitor group members, [A6.2] Manage performance goals and [A6.3] Provide feedback.

Monitor group members. The participants recommended that two requirements should be considered when designing the group monitoring system administered by the moderator. The first design requirement is that the moderator is able to monitor group members' performances [A6.1.1] and require the moderator should be able to "monitor group member's progress and achievement and compare it with other group members". The second requirement is that the moderator should have the ability to monitor the group's communication style and interactions, such as being a distraction or helper [A6.1.2]. Monitoring members' interactions such as "monitor messages and feedback send between members", the interaction could be positive or annoying messages.

Manage performance goals. The participants highlighted the importance of setting goals and considered it as one of the moderator's tasks and they suggested various opinions to the moderator when managing performance goals. The first opinion recommended moderator should specify performance goals for members [A6.2.1] and the participants suggested the online peer group platforms should be designed to "allow moderators to set goals specifically for new members". Some of the participants mentioned that some of the group members had difficulty controlling their behaviour and they required "more help and support from moderators, specifically in setting their own goals". 
The second viewpoint was that the moderator should be able to modify goals for members [A6.2.2]. The participants suggested two various ways of modifying the goals. The first viewpoint is that the moderator should be able to stop the tracking system for specific users for a period. For example, a participant said that "I am a student and sometimes I use digital media for study purposes, so it is useful if the moderator enables me to stop the tracking system, after contacting the moderator and clarifying a reasonable reason". The second viewpoint is the platform should be designed to enable the moderator to change the time-plan for a specific user. For example, a participant mentioned that "sometimes I am doing something important or I am travelling so I need extra time on specific days' the moderator should be able to change the time plan".

The third requirement is that the moderator should be able to review individual and collective goals [A6.2.3] and have the ability to modify the goals to become achievable goals that would motivate users to commit to the group goals. The fourth opinion is that the moderator should have the ability to discuss with members the "barriers to goal attainment" [A6.2.4]. The last opinion is moderator should be able to send personalised best practices and advice on how to achieve goals to members [A6.2.5].

Provide feedback. The participants suggested various design requirements of the moderator feedback; also, they recommended several features to moderator feedback which included reason and subject of the feedback, communication channesl and feedback framing. The interview analysis indicated that participants required various requirements about the feedback's reasons and subject, the first requirement is to send feedback about how the group is performing [A6.3.1]. The moderator feedback should be based on "compare peer progress with a specific peer" that has a similar profile or demography. Participants argue that peer-to-peer progress feedback would help them to know their progress level and identify if their progress is good, or if they or need to work more to achieve the group goals.

The second requirements suggested moderator provide feedback about self-progress to members [A6.3.2]. The participants recommended that the moderator feedback should be based on "compare current performance and past-performance which would help to know the progress and would encourage achieving the usage goals". Moreover, participants felt that the moderator feedback is not only about user performance and progress; they recommended that the feedback should involve member's interaction within the group [A6.3.3].

The fourth requirement is that the moderator should choose the communication channel to use with members [A6.3.4]. Participants suggested different communication preferences and moderator feedback, such as receiving feedback as writing, non-verbal cues such as emoji's, pop-up text messages or in chatrooms, whether audio or text. The last requirement is that the moderator should choose the framing and the tone of the feedback [A6.3.5]. Participants mentioned two types of framing in which is a positive frame or frame the feedback to have "an order and gaudiness". Also, the participants mentioned the importance of feedback tone; they suggested that the feedback tone should be "strict and more formal". In their opinion, strict feedback should be more effective and forced to follow the moderator guide and advice. 


\section{Moderation requirements: personal and cultural effects}

A series of linear multiple regressions using the enter method were conducted on the survey results. In each model the predictors were gender (male/ female); country (UK/ Middle East); perceived usefulness of peer support groups; willingness to join a peer support group; the five personality trait scores of extraversion, agreeableness, conscientiousness, neuroticism and openness; and finally, the self-control score. For each model, the outcome measure was the individual questions used to measure attitudes relating to the moderator role and tasks, as identified within the description of each model result in the section below. Multicollinearity diagnostic was conducted prior to the analysis to determine the suitability of conducting multiple regressions.

\subsection{Moderator nature [A1]}

In term of the nature of the moderator models, we found that none of the models was significant for the three outcome measures relating to this topic were significant, which were [A1.1] Software, e.g. automatic target calculation and advice-giving; [A1.2] Human; or [A1.3] Blended, i.e. human and software together.

\subsection{The strategy for allocating human moderators [A2]}

The strategy for allocating group moderator has four models; the regression analysis indicated that one of the models tested within this section was significant. The findings showed that [A2.1] voting by group members is a significant model for allocating the group moderator. Voting by members significantly predicted $12 \%$ of the variance $\left(\mathrm{R}^{2}\right.$ $=.12, \mathrm{~F}(10,160)=2.12, \mathrm{p}<.05)$, with the significant predictors being perceived usefulness of online peer support groups $(\beta=-.21)$, willingness to join online peer support groups $(\beta=.25)$, openness $(\beta=-.09)$ and self-control $(\beta=-.03)$. As such as perceived usefulness of online peer support groups increased if the agreement with the strategy of voting by members increased; however conversely willingness to join online peer support groups decreased as an agreement with this strategy increased. As both openness and self-control increase is a decrease as acceptance of the strategy decreases. The models for the three remaining strategies ([A2.2], [A2.3] and [A2.4]) within this section were non-significant.

\subsection{Moderator skills [A3]}

In term of the moderator skills models, we found that none of the models was significant for the three outcome measures relating to this topic, which were [A3.1] Had the well-being issue themselves in the past and recovered from it; [A3.2] High communication skills (verbal and non-verbal, diplomacy, motivating language, etc.); [A3.3] Knowledge, e.g. behavioural change, management and leadership skills. 


\subsection{Moderator authority $[\mathrm{A} 4]$}

In term of the moderator authority in the peer group, the analysis found that one of the models within the section was significant. The model for [A4.1] Manage membership, e.g. adding new members and banning members who violate the rules, etc. $\left(\mathrm{R}^{2}=\right.$ $.15, \mathrm{~F}(10,160)=2.74, \mathrm{p}<.01)$ accounted for $15 \%$ of the variance was significantly predicted by the single predictor of conscientiousness $(\beta=.19)$. As such acceptance of this strategy increased as conscientiousness increased. The other two models ([A4.2] and [A4.3]) were not significant.

\subsection{Ability and responsibility to apply reinforcement functions [A5]}

The responsibility of the moderator to issue rewards and penalties has four models. The regression analysis finding showed that two of the models in this section were significant. The outcome of [A5.1] Rewards to members based on the improvement of their performance model $\left(\mathrm{R}^{2}=.15, \mathrm{~F}(10,160)=2.19, \mathrm{p}<.05\right)$ accounted for $15 \%$ of the variance and was significantly predicted by agreeableness $(\beta=.09)$, conscientiousness $(\beta=.12)$ and self-control $(\beta-.03)$. As such acceptance of this strategy increases as does agreeableness and conscientiousness; however, as self-control increases acceptance of the strategy decreases. The outcome of [A5.3] Rewards based on the member's interactions within the online group, e.g. helping others, etc. model $\left(\mathrm{R}^{2}=.11, \mathrm{~F}(10,160)=\right.$ $1.98, \mathrm{p}<.05)$ accounted for $11 \%$ of the variance and was significantly predicted equally by agreeableness $(\beta=.10)$ and conscientiousness $(\beta=.10)$. The other two models within this section ([A5.2] and [A5.4]) were not significant.

\subsection{Moderator tasks [A6]}

[A6.1] Moderator ability and responsibility to monitor the group members. One of the moderator tasks is to monitor the group members, which was analysed with two models. The regression analysis findings show that one model in this section was significant. The finding showed that [A6.1.2] Access data around the style of communication of members, e.g. reports indicating members to be helpful, distractor, digression, etc. model $\left(\mathrm{R}^{2}=.12,(\mathrm{~F}(10.160)=2.222, \mathrm{p}<.05)\right.$ accounted for $12 \%$ of the variance, with two predictors significantly contributing to the model: conscientiousness $(\beta=.13)$, and self-control $(\beta=-.03)$. As such acceptance of this strategy increased as conscientiousness increased; however, as self-control increases acceptance of the strategy decreases. The other model in this section [A6.1.1] was not significant.

[A6.2] Moderator ability and responsibility to manage performance goals. The responsibility of the moderator to manage performance goals has five models. The regression analysis showed that three models in this section were significant. The model [A6.2.1] specify performance goals for members $\left(\mathrm{R}^{2}=.12, \mathrm{~F}(10,160)=2.317, \mathrm{p}<.05\right)$ accounted for $12 \%$ of the variance and was significantly predicted by conscientiousness $(\beta=.08)$, and self-control $(\beta=-.02)$. As such acceptance of strategy increased as conscientiousness increased, however, as self-control increases acceptance of the strategy decreases. The outcome of [A6.2.3] The strategy of Review goal achievement with 
members frequently $\left(\mathrm{R}^{2}=.12, \mathrm{~F}(2.233), \mathrm{p}<.01\right)$ accounted for $12 \%$ of the variance and was significantly predicted by the willingness to join online peer support groups $(\beta$ $=.19)$, conscientiousness $(\beta=.09)$ and neuroticism $(\beta=.07)$. As such as acceptance of strategy increased as the willingness to join online peer support groups and neuroticism increased; however, respondents from the Middle East were significantly less likely to demonstrate acceptance of this strategy. The other three models within this section were not significant, which had the outcomes of [A6.2.2], [A6.2.4] and [A6.2.5].

[A6.3] Moderator responsibility and permission to provide feedback to members. In term of the moderator provide feedback, the analysis found that two models within section were significant. The model for [A6.3.2] Send feedback about self-progress to members, e.g. their self-improvement $\left(\mathrm{R}^{2}=.12, \mathrm{~F}(10,160), \mathrm{p}<.05\right)$ accounted for $12 \%$ of the variance was significantly predicted by the single predictor of self-control $(\beta=$ 0.025). As such acceptance of this strategy increased as does self-control decreased. The model for [A6.3.4] Choose the communication channel to use with members, e.g. text, audio, non-verbal such as emoji, chat, etc. was significant $\left(\mathrm{R}^{2}=.15, \mathrm{~F}(10,160)=\right.$ $2.712), \mathrm{p}<0.05$ ), accounting for $15 \%$ of the variance, significantly predicted by the three predictors. These were culture, extraversion and openness $(\beta=-.49),(\beta=-.13)$, $(\beta=-.11)$. As such, both extraversion and openness increased, acceptance of the strategy decreases. There was significantly greater acceptance of this strategy in the UK than in the Middle East.

The model for [A6.3.5] Choose the framing and the tone of the feedback, e.g. guidance, assertive, strict, friendly, etc. was significant $\left(\mathrm{R}^{2}=.14, \mathrm{~F}(10,160), \mathrm{p}<.01\right)$, accounting for $14 \%$ of the variance of the model. This was predicted by culture and selfcontrol $(\beta=-.29)$ and $(\beta=-.31)$. As such acceptance of this strategy increased as selfcontrol decreased, and the strategy was significantly more likely to be accepted in the UK than the Middle East. Both models for [A6.3.1] and [A6.3.3] were not significant.

\section{Discussion}

The regressions that were found to be significant accounted for approximately 12 $15 \%$ of the variance in each outcome measure. As such, they in part, explain the reported attitudes and opinions, albeit it to a relatively small degree. The two personality traits of agreeableness and conscientiousness, along with self-control, were consistently amongst the significant predictors. This is perhaps as would be expected, as each of these predictors can feasibly relate to how accepting an individual is to be part of a group and to have their actions shaped and monitored by members in that group.

It is interesting to note which predictors were not found to be significant. The model for a preference towards the moderator being human, software or a combination of both was not significant. This is in contrast to various models of technology acceptance, from which it could be expected that individuals may not respond to technology-based agents in the same way as human group members [1]. In addition, it has been found consistently throughout social psychological research that individuals exhibit biases 
when comparing their own knowledge and skills against those of their peers (for example [2]). In relation to the results of the current study, this may suggest that people do not distinguish between a moderator who is human and one who is software-based. Research into the leader-member exchange theory in a range of domains, including health information management [3], has demonstrated that the relationship between a leader and a group is complex, with expectations on the part of the group members on how the group should be managed. Despite this, the regression models for moderators' skills (knowledge, leadership communication) were also not significant. Given this, it is of interest that none of the predictors appeared to differentiate between human and software-based group moderators.

The model for moderator allocation occurring through a voting system was significant, although it is notable that whilst the perception of the usefulness of this strategy was a positive predictor, the willingness to join such a group was a negative predictor. This may suggest that individuals recognise the benefits of the democratic process of choosing the moderator through an election, without necessarily wishing to be subject to the consequences of this voting process. This could relate to the need to assert uniqueness, which refers to the drive individuals have to demonstrate that they are not bound by social rules [4]. This could reflect an awareness of the part of the respondents of the phenomena of groupthink, in which groups are observed to make more risky, extreme and often objectively worse decision than individuals do alone [5]. Avoiding unintended consequences such as these are of course one of the underlying reasons why the group would have a moderator in the first instance; however, given the relative novelty of moderator facilitated online peer support groups it is possible that when asked about this respondents struggled to conceptualised what was meant.

Social facilitation occurs when the presence of an audience improves performance, as explained by drive theory [6].This may result in part account for the significant regression model which found that the personality traits of agreeableness and conscientiousness positively predicted the acceptance of rewards within the group, although it was also noted that increased self-control appeared to reduce the acceptance of this strategy. This suggests that there is a trade-off between the willingness to engage in this strategy and the desire to maintain personal independence and control. Related to this is evaluation apprehension, in which performance is negatively impacted by the presence of others [7].This may account for the non-significant regressions models relating to applying penalties to members for poor performance, i.e. individuals may be receptive of the concept of group monitoring provided that this is not associated with evaluation or punishment. This is in keeping with previous research, which suggests that whilst group membership typically decreases evaluation apprehension, this only occurs when the individual knows they will not be scrutinised individually [8]. In the case of online peer support groups individuals may perceive that their actions are highly quantifiable and traceable, leading to an increase in evaluation apprehension.

There is limited research on the relationship between personality and preferred group moderation characteristics in either online or offline settings. There is though some research on personality and management styles within organisation that are of relevance to this study, such as for example [9], which found that openness and conscientiousness 
contributed towards group performance, when managed appropriately. Both personality traits were significant predictors in several of the regression models conducted within this study. However, several other personality traits were not significant predictors in any of the models. This includes neuroticism, which refers to the tendency to experience emotions such as anxiety, fear, frustration and loneliness. Given the nature of the proposed online peer support groups, and the aforementioned possibility of phenomena such as apprehension evaluation occurring, it is odd that this personality trait was not a significant predictor.

The overall pattern of results was reflected in relation to the tasks, responsibilities and powers of the group moderator, with the significant predictors with the significant models typically including self-control and the personality trait of conscientiousness. These results can be considered in relation to research into power and group dynamics in groups. It has been noted, for example, that group members expect those with a leadership role within a group to adhere to the social norms of that group [10]. This reflects the comments made by participants that they would accept rules and permissions determined by the moderator, provided that these are transparent and fair. It was found that participants from the UK were more likely to wish to have control over how these tasks and permissions were controlled. This is consistent with research from Hofstede Insights, which suggests that the UK scores are higher on individualism and power distance as compared to the KSA and Syria [11]. This relationship between culture and power within-group leadership roles has, however, been found to be a complex one, with for example, leaders who violate norms in individualistic cultures being viewed as more powerful. Similarly, identification with the group has been found to be associated with a greater sense of responsibility for the wellbeing of the group [12]. This highlights the importance of those individuals who have the power within the group having an investment in that group. Finally, it was noted that some participants stated a preference for direct and authoritarian styles of communication from the moderator provided that, as noted previously, this did not violate the expectations and social norms of the group. It has been observed within sports psychology research that whilst prescriptive and authoritarian approaches to behaviour change are increasingly seen as outdated they can in some contexts nevertheless still be effective, particularly in relation to deviation from desired behaviour [13].

Previous research into gender would suggest the males and females make different use of social support networks to manage behaviour change [14]; including within internet support groups [15]. This was not found to be the case in this study, as gender was not a significant predictor in any of the significant regression models. Similarly, culture was not a significant predictor in the majority of models. .These are both factors which could be expected to impact on attitudes towards peer group hierarchies and purposes, and so it is of interest that they appeared to be of relatively little importance with regards to online behaviour change peer support groups in this study. Again there is a lack of research on this particular topic, with little understanding of how group dynamics are influenced by cultural factors [16]. This may reflect a criticism that has been made of psychological research, which it relies too heavily upon samples from Western countries [17]. The research reported in this study contributes to reducing this gap. 
Overall several predictors within the regression models reported in this study should, as based on previous psychological research, be reasonably expected to significantly predict attitudes towards the moderation of online peer groups. The fact that they did not is important, both for our theoretical understanding and for the practical implementation of such systems. As identified by [18] with reference to the online disinhibition effect, there is a question over whether the internet enhances or transforms; that is whether it causes people to behave in fundamentally different ways when online, or if it enhances pre-existing traits and processes. This is not a question that has been definitively addressed within the research literature and, as this study illustrates, is something we must investigate further if effective and appropriate behaviour change systems are to be developed.

\section{Conclusion}

In this paper, we extended our work in [36], where we studied the acceptance and rejection factors of online peer support groups and investigated the role and tasks of the moderator of such groups. Such groups are purpose-driven social networks which are meant to encourage and boost positive behaviour and prevent relapse. We did the investigation qualitatively and quantitatively with people who self-declared to have wellbeing issues. The understanding of their views is meant to help the design of the online platforms that host peer support groups in Human-Centered Design (HCD) approach. For example, it helps in the decision of the tools to make available to moderators to manage the groups in facets like memberships and rewards. It also helps governance processes and common grounds formation, e.g. in the allocation of moderators and their management style. Tailoring the group moderation settings correctly can help to prevent negative side effects such as members' reactance and lowering their self-esteem and to increase commitment to groups and their mission. Finally, this study contributes to the literature by helping the elicitation and customisation of the requirements and design of social behaviour change tools, mainly on what moderation aspects to be studied and analysed and fitted to the application domain.In the wider context, this study is meant towards an interdisciplinary systems analysis and design where social sciences and psychology support software engineering processes, especially where mistakes in the design can lead to negative behaviour and cause harm to users. Most commercial apps around behaviour change seem to lack theory-informed design. They are mainly focused on usability and attractiveness and seem to apply engagement elements, such as gamification, in ad-hoc style instead of robust evidence and established theories.

\section{References}

1. Taherdoost, H.: A review of technology acceptance and adoption models and theories. Procedia Manufacturing (22), 960-967(2018).

2. Olson, J.M., Ross M.: False feedback about placebo effectiveness - consequences for the misattribution of speech anxiety. Journal of Experimental Social Psychology. 24(4), 275291(1988). 
3. Hunt, T.J.: Leader-member exchange relationships in health information management. Perspectives in health information management (11) Spring,(2014).

4. Imhoff, R. Erb H.P.: What motivates nonconformity? Uniqueness seeking blocks majority influence. Personality and Social Psychology Bulletin, 35(3),309-320 (2009).

5. Schafer, M. Crichlow S.: Antecedents of groupthink: A quantitative study. Journal of Conflict Resolution, 40(3), 415-435(1996).

6. Zajonc, R.B., Social facilitation. Science,(149), 269 - 274 (1965).

7. Platania, J.Moran G.P.:Social facilitation as a function of the mere presence of others. J Soc Psychol,141(2),190-197(2001).

8. Crisp, R.J. Turner R.N.: Essential social psychology. 4th Edition. ed., Thousand Oaks: SAGE Publications India Pvt Ltd. pages cm (2017).

9. Dai, S.L., Y.C. Li, Zhang W.: Personality traits of entrepreneurial top management team members and new venture performance. Social Behavior and Personality, 47(7),15 (2019).

10. Stamkou, E., van Kleef G.A., Homan A.C.: Feeling entitled to rules: Entitled individuals prevent norm violators from rising up the ranks. Journal of Experimental Social Psychology, 84(1).10, (2019).

11. Hofstede Insights. Compare countries( 2019). https://www.hofstede-insights.com/product/compare-countries/Accessed 28 April 2019.

12. Scholl, A.,Sassenberg K, Ellemers N, Scheepers D, De Wit F.: Highly identified powerholders feel responsible: The interplay between social identification and social power within groups. British Journal of Social Psychology, 57(1),112-129 (2018).

13. Delrue, J., Soenens B, Morbée S, Vansteenkiste M, Haerens L.:Do athletes' responses to coach autonomy support and control depend on the situation and athletes' personal motivation? Psychology of Sport and Exercise (43), 321-332(2019).

14. Matud, M.a.P., Ibañez I, Bethencourt JM, Marrero R, Carballeira M.: Structural gender differences in perceived social support. Personality and Individual Differences,. 35(8),19191929 (2003).

15. Strom, J., Høybye MT, Laursen M, Jørgensen LB, Nielsen CV.: Lumbar spine fusion patients' use of an internet support group: Mixed methods study. Journal of Medical Internet Research, 21(7), 17 (2019).

16. van Zomeren, M. Louis W.R.: Culture meets collective action: Exciting synergies and some lessons to learn for the future. Group Processes \& Intergroup Relations,20(3), 277-284 (2017).

17. Henrich, J., Heine S.J., Norenzayan A.: The weirdest people in the world? Behavioral and Brain Sciences, 33(2-3), 61-83(2010).

18. Suler, J.: The online disinhibition effect. Cyberpsychology \& Behaviour, 7(3), 321-6 (2004).

19. Ciocarlan, A., Masthoff, J.Oren, N.: July. Kindness is contagious: Study into exploring engagement and adapting persuasive games for wellbeing. In Proceedings of the 26th Conference on User Modeling, Adaptation and Personalization, 311-319 ACM (2018).

20. Rajani, N.B., Weth, D., Mastellos, N. and Filippidis, F.T.: Use of gamification strategies and tactics in mobile applications for smoking cessation: a review of the UK mobile app market. BMJ open, 9(6), (2019).

21. McKay, F.H., Wright, A., Shill, J.: Stephens, H. Uccellini, M.:Using health and well-being apps for behavior change: a systematic search and rating of apps. JMIR mHealth and uHealth, 7(7),(2019).

22. Alrobai, A., Algashami, A., Dogan, H., Corner, T., Phalp, K., Ali, R., COPE.er Method: Combating Digital Addiction via Online Peer Support Groups. International journal of environmental research and public health, 16(7),1162(2019) 
23. Braun, V., Clarke, V. and Terry, G.: Thematic analysis. Qual Res Clin Health Psychol, 24, 95-114 (2014).

24. Matthews, J., Win, K.T.,Oinas-Kukkonen, H. Freeman, M.:Persuasive technology in mobile applications promoting physical activity: a systematic review. Journal of medical systems, 40(3), 72 (2016)

25. Orji, R. and Mandryk, R.L.: culturally relevant design guidelines for encouraging healthy eating behavior. International Journal of Human-Computer Studies, 72(2),207-223 (2014).

26. Orji, R., Mandryk, R.L. and Vassileva, J.: Gender, age, and responsiveness to Cialdini's persuasion strategies. In International Conference on Persuasive Technology ,147-159 (2015) Springer, Cham.

27. Alkış, N. and Temizel, T.T.:The impact of individual differences on influence strategies. Personality and Individual Differences,(87),147-152 (2015).

28. Ciocarlan, A., Masthoff, J. and Oren, N.: Kindness is Contagious: Exploring Engagement in a Gamified Persuasive Intervention for Wellbeing. In PGW@ CHI PLAY (2017).

29. Lowensteyn, I., Berberian, V., Berger, C., Da Costa, D., Joseph, L. and Grover, S.A.:. The Sustainability of a Workplace Wellness Program That Incorporates Gamification Principles: Participant Engagement and Health Benefits After 2 Years. American Journal of Health Promotion, (2019).

30. Cialdini, R. Influence: The Psychology of Persuasion.

31. Rammstedt, B. and John, O.P.: Measuring personality in one minute or less: A 10-item short version of the Big Five Inventory in English and German. Journal of research in Personality, 1 (41),203-212 (2007).

32. Tangney, June P., Roy F.: Baumeister, and Angie Luzio Boone. "High self-control predicts good adjustment, less pathology, better grades, and interpersonal success. Journal of personality 2 (72), 271-324 (2004).

33. Maro, S., Sundklev, E., Persson, C.O., Liebel, G. and Steghöfer, J.P., 2019, March. Impact of Gamification on Trace Link Vetting: A Controlled Experiment. In International Working Conference on Requirements Engineering: Foundation for Software Quality (REFSQ'19). pp. 90-105). Springer, Cham.

34. Barn, B.S. and Barn, R., 2018, June. Human and value sensitive aspects of mobile app design: a Foucauldian perspective. In International Conference on Advanced Information Systems Engineering (CAiSE'18). pp. 103-118. Springer, Cham.

35. Haake, P., Kaufmann, J., Baumer, M., Burgmaier, M., Eichhorn, K., Mueller, B. and Maedche, A., 2018, June. Configurations of User Involvement and Participation in Relation to Information System Project Success. In International Conference on Advanced Information Systems Engineering (CAiSE’18). pp. 87-102. Springer, Cham.

36. Aldhayan, M., Cham, S., Kostoulas, T., Almourad, M.B. and Ali, R., 2019, April. Online Peer Support Groups to Combat Digital Addiction: User Acceptance and Rejection Factors. In World Conference on Information Systems and Technologies (pp. 139-150). Springer, Cham. 